\title{
Finite element modelling of the instability in rapid fracture of graphene
}

\author{
Bin Zhang ${ }^{\mathrm{a}, *}$, Haifeng Xiao ${ }^{\mathrm{a}}$, Gang Yang ${ }^{\mathrm{a}}$, Xiaoming Liu ${ }^{\mathrm{b}}$ \\ ${ }^{a}$ State Key Laboratory of Mechanics and Control of Mechanical Structures, and College of Aerospace Engineering, Nanjing University of Aeronautics \\ and Astronautics, Nanjing 210016, China \\ ${ }^{\mathrm{b}}$ LNM, Institute of Mechanics, Chinese Academy of Sciences, Beijing 100190, China
}

\section{A R T I C L E I N F O}

\section{Article history:}

Received 6 May 2014

Received in revised form 7 May 2015

Accepted 10 May 2015

Available online 18 May 2015

\section{Keywords:}

Dynamic fracture

Graphene

Crack velocity

Finite element

\begin{abstract}
A B S T R A C T
A finite element model of graphene strip is developed to predict the instability of dynamic fracture, in which the $\mathrm{C}-\mathrm{C}$ bonds are represented by Timoshenko beam elements, and the constitutive relation of beam is derived from the atomic potential. Crack instability and branching are observed under loading strain rates from $\sim 10^{-5} \mathrm{fs}^{-1}$ to $\sim 10^{-8} \mathrm{fs}^{-1}$. Under low loading rates, the initiated midway crack propagates straight at supersonic velocity, while kinking and oscillation occur beyond a critical crack velocity $\sim 10.33 \mathrm{~km} / \mathrm{s}$ under high rates. The results demonstrate that our equivalent models could provide efficient information for studying the fracture in graphene.
\end{abstract}

(c) 2015 Elsevier Ltd. All rights reserved.

\section{Introduction}

Graphene has triggered significant interests for its two-dimensional (2D) hexagonal crystal structure and ultrahigh mechanical strength [1,2]. With graphene being developed as the candidate of toughening nano-composites [3], the fracture mechanical behaviours, especially under dynamic loading conditions, are drawing the attention of researchers [4,5]. Although pristine graphene has exceptional high strength, defects (e.g. cracks) and grain boundaries are inevitable during the exfoliation of graphene samples, which could lower the strength and fracture toughness largely. In theoretical modelling, Le et al. [4] studied edge crack growth in graphene under simple tension using molecular dynamics (MD) simulations, and reported that the crack speed increases with the time step and decreases with the initial crack length. Zhang et al. [5] investigated the instability of crack motion in graphene by atomistic MD simulations, which showed that the brittle crack along zigzag (ZZ) direction branches around $8.20 \mathrm{~km} / \mathrm{s}$, equivalent to 0.65 of Rayleigh-wave speed in graphene, and validated theoretical predictions of rapid fracture instability in elastodynamics [6]. Omeltchenko et al. [7] also probed the fracture velocity of graphene by MD modelling based on the Tersoff-Brenner potential. Wang et al. [8] studied the fracture behaviours of single-layered graphene sheets with edge cracks under simple tension by MD, and stated that cracks propagate faster in higher strain rates. Zhao et al. [9] showed that temperature has an important effect on fracture strength of graphene. Xu et al. [10] proposed a coupled quantum/continuum mechanics approach to study crack propagation in graphene, and the critical stress intensity factors (SIFs) were calculated to be $4.21 \mathrm{MPa} \sqrt{ } \mathrm{m}$ and $3.71 \mathrm{MPa} \sqrt{ } \mathrm{m}$ in $\mathrm{ZZ}$ and armchair (AC) sheets respectively. In experiments, Kim et al. [11] explored the tearing process of suspended monolayer graphene membranes by high-resolution transmission electron microscopy, and showed that the tearing angle changes occasionally by $30^{\circ}$ along

\footnotetext{
* Corresponding author. Tel.: +86 13913811289; fax: +86 2584891422.

E-mail addresses: beenchang@nuaa.edu.cn, been.chang@gmail.com (B. Zhang).
} 


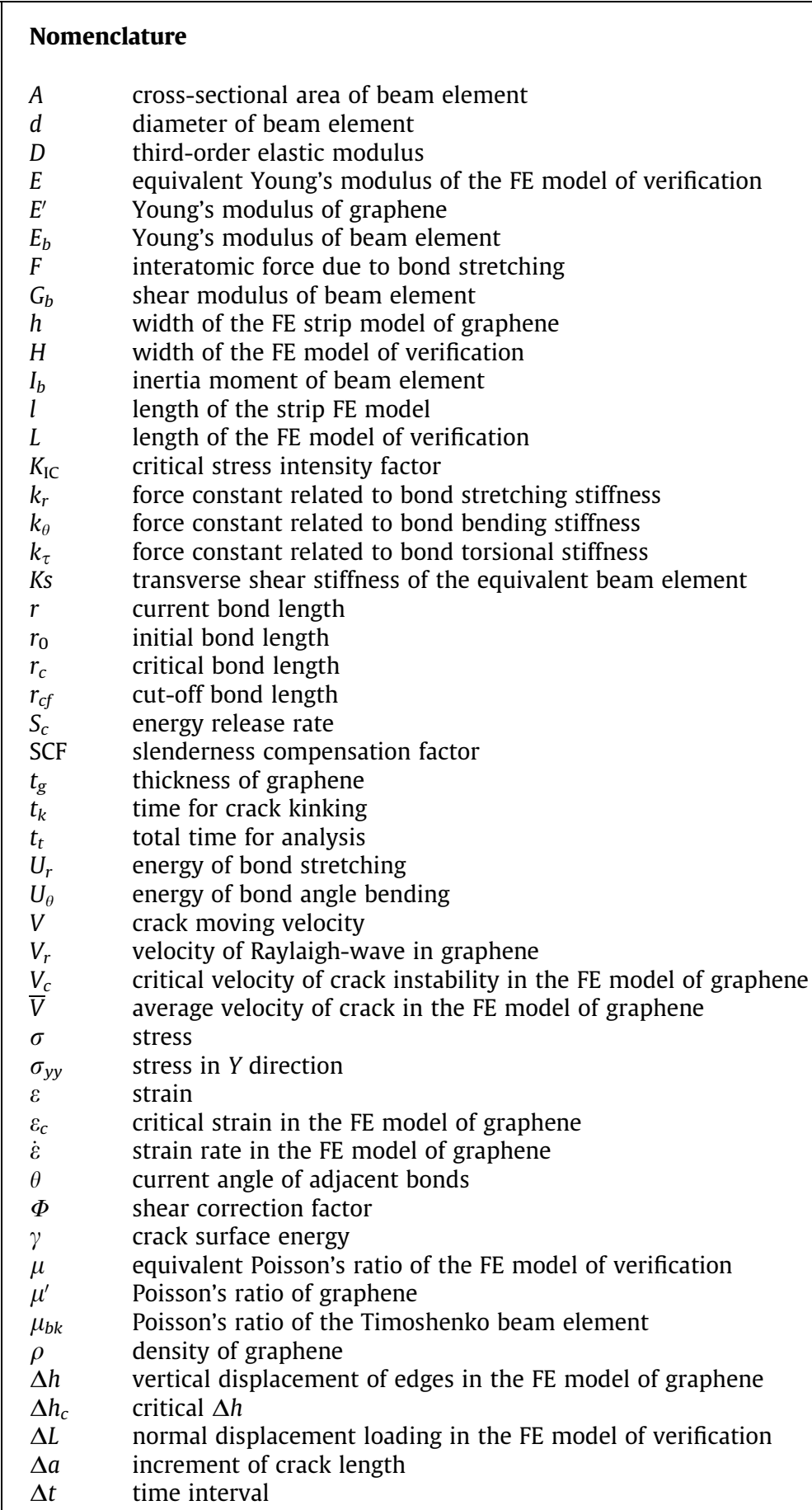

AC or ZZ edges. Lee et al. [12] reported the initiation angle of radial cracks of multilayer graphene by using miniaturized ballistic tests, and found that the distribution of the angle between adjacent cracks displays preferences for small multiples of $30^{\circ}$. Therefore, it would be interesting to examine the characteristics of dynamic fracture in graphene, as a layer reinforcer, to prevent catastrophic failure in composite engineering. 
Alternatively, equivalent finite element (FE) models based on continuum theory can overcome the limit of calculation scales in atomic simulations. Li et al. [13] ever developed a structural mechanics approach according to the similarity of geometry and energy expression form between $\mathrm{C}-\mathrm{C}$ bonds and beams to investigate elastic properties of single wall carbon nanotubes (SWCNTs). To imitate covalently bonding in low-dimensional carbon materials by equating the harmonic potential of bonds with the deformation energy of beams, equivalent macro models can be constructed and analyzed based on classical structural mechanics. Recently, some researchers [14-17] attempted to use FE methods to study nonlinear or fracture mechanical behaviours of nano-materials by the molecular structural mechanics approach, e.g. Tserpes et al. [14] proposed an atomistic-based progressive fracture model to simulate the fracture behaviours of SWCNTs using ANSYS, where non-linear characteristic of Euler-Bernoulli (EB) beam elements was obtained from the modified Morse potential. Based on the fracture FE model of Tserpes et al. [14], Baykasoglu et al. [15] analyzed stress-strain behaviours of pure and defective monolayer graphene along $\mathrm{AC}$ and $\mathrm{ZZ}$ directions respectively, and simply explored the crack propagation properties by MATLAB. Parashar and Mertiny [17] investigated the mode-I fracture properties of graphene by an FE-based atomistic model, and estimated the strain energy release rate.

To our knowledge, the instability of crack propagation in graphene has rarely been studied by FE methods. Therefore, we here will construct an equivalent FE model to study dynamic fracture of brittle cracks along ZZ direction in a strip model of graphene under mode-I displacement loading, in which large deformation and nonlinear bonding interaction are considered. The crack velocities, critical SIFs, critical energy release rates and other fracture properties under various strain rates are analyzed afterwards.

\section{FE model of graphene for dynamic fracture study}

\subsection{Equivalent nonlinear Timoshenko beams for C-C bonds}

The Timoshenko beams as representative models, enabling deep shear cross-deformations [18], are suitable to consider realistic distributions of thickness and equilibrium length of $\mathrm{C}-\mathrm{C}$ bonds and the covalent bond breakage with nonlinear geometric effects. Conversely, slender EB beam elements, ever used by both Tserpes et al. [14] and Baykasoglu et al. [15] to simulate $\mathrm{C}-\mathrm{C}$ bonds' large deformations and nonlinear geometric effects, cannot withstand transverse shear deformation and can only be theoretically used for small-strain and large-rotation analysis for the cubic interpolation functions [19]. Therefore, the $\mathrm{C}-\mathrm{C}$ bonds in graphene are equated by nonlinear Timoshenko beams in this paper, which are represented by the linear planar beam elements (Beam 21) in Abaqus 6.9.

We here employ the constants $k_{r}=84.7 \mathrm{nN} \AA^{-1}$ [20], $k_{\theta}=9.00 \mathrm{nN} \AA \mathrm{rad}^{-2}$ and $k_{\tau}=2.78 \mathrm{nN} \mathrm{Arad}^{-2}$ [21] for equivalent Timoshenko beams of $\mathrm{sp}^{2} \mathrm{C}-\mathrm{C}$ bonds accordingly (definitions of prefactors can be found therein) because the tensile force-displacement curves obtained by either the modified Morse potential or the Tersoff-Brenner potential yield the same before the inflection point [20]. The constants of beam elements are obtained and represented in Table 1 .

Since the B21 element could endure stretching deformation and transfer shearing deformation during the whole analysis procedure, here we mainly consider nonlinear effects of the beam length $l$ [22], and determine the reasonable values of the shear correction factor $\Phi$ and transverse shear stiffness $K s$ which are fixed during the large deformation of the beam element [19]. When the toggle "Nlgeom" is on, Abaqus/Explicit will account for geometric nonlinearity during the step. $\Phi$ is represented by the slenderness compensation factor $(S C F)$. SCF and $K s$ of beam elements are 0.5 and $160 \mathrm{GPa}^{2} \mathrm{~nm}^{2}$ respectively, which are determined by a trial and error procedure to guarantee that the Young's modulus and Poisson's ratio of graphene FE model (see Section 2.2) are close to their experimental values [1] as far as possible.

Under in-plane deformations without torsion for graphene, total potential energy can be written as $U_{\text {total }}=\Sigma U_{r}+\Sigma U_{\theta}$, where $U_{r}$ and $U_{\theta}$ are the bonding energy due to bond stretching and bending respectively, and $r$ is the current bond length. As only the stretching energy $U_{r}$ dominates the fracture behaviours of graphene [15,16], $U_{\theta}$ (responsible for stabilizing the molecular structure) is neglected afterwards.

At room temperature, the atomic scale fracture process in graphene is governed by brittle breaking of atomic bonds at crack tips [23]. Belytschko et al. [20] studied the fracture of CNTs by molecular mechanics simulations, and reported that the fracture of $\mathrm{C}-\mathrm{C}$ bonds depends primarily on the interatomic potential before the inflection point, after which, the form of the potential function is no more important as material damage occurs. Once the length of a $\mathrm{C}-\mathrm{C}$ bond exceeded a critical distance $r_{c}$, the interatomic force would drop rapidly to zero, and the bond is regarded as broken. This fracture criterion for C-C bonds can be realized by distanced-based detection method [24].

Table 1

Constants of B21 element [18].

\begin{tabular}{ll}
\hline Bond cross-sectional diameter, $d$ & $0.89 \AA$ \\
Bond length, $r_{0}$ & $1.42 \AA$ \\
Cross-sectional area, $A$ & $0.622 \AA^{2}$ \\
Moment of inertia, $I_{b}$ & $0.0308 \AA^{4}$ \\
Young's modulus, $E_{b}$ & $19.5 \mathrm{TPa}$ \\
Poisson's ratio, $\mu_{b}$ & 0.23 \\
Shear modulus, $G_{b}$ & $7.93 \mathrm{TPa}$ \\
\hline
\end{tabular}


As an accurate atomistic interaction expression, the Tersoff-Brenner potential [25] is used to simulate the fracture of graphene, which involves large deformations and geometric nonlinear effects of $\mathrm{C}-\mathrm{C}$ bonds. For each $\mathrm{C}-\mathrm{C}$ bond, the Tersoff-Brenner potential is employed before its fracture, after which the broken $\mathrm{C}-\mathrm{C}$ bonds (beam elements) disappear (are deleted), namely, fracture evolution of the whole model is shown by the successional breakages of bonds (beams). Many-body interactions of atoms (nodes) are not considered here. Accordingly, we derived the nonlinear constitutive relation of B21 element from the stretching portions of the Tersoff-Brenner potential. To avoid unphysical spurious bond forces [5], the interaction range of carbon atoms is considered only within a distance $r_{c}=1.7 \AA$. Therefore, the cut-off distance $1.7 \AA$, which corresponds to the inflection point (i.e., the peak force) at approximately $19.7 \%$ strain, is employed. The bond-stretching energy $U_{r}$ is then as

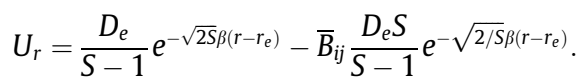

The interatomic force versus bond length for a single $\mathrm{C}-\mathrm{C}$ bond in graphene is obtained

$$
F=\frac{\partial U_{r}}{\partial r}=D_{e} \beta \frac{\sqrt{2 S}}{S-1}\left[-e^{-\sqrt{2 S} \beta\left(r-r_{e}\right)}+\bar{B}_{i j} e^{-\sqrt{2 / S} \beta\left(r-r_{e}\right)}\right],
$$

where the potential parameters $D_{e}=6.0 \mathrm{eV}, \beta=2.1 \AA^{-1}, S=1.22, r_{e}=1.39 \AA$ [25].

Moreover, the second generation version of the Tersoff-Brenner potential [26] considers the effect of the third class distance atoms by adding a term $B_{i j}^{\pi}$ to $\bar{B}_{i j}$, which leads to the initial length of C-C bond changing from $1.45 \AA$ to $1.42 \AA$ [27]. Thus, the modified $\bar{B}_{i j}$ is obtained by assuming $F_{r=1.42 \AA}=0$, and the $F-r$ curve is obtained and shown in Fig. 1 . By use of $\sigma=F / A$ and $\varepsilon=\left(r-r_{0}\right) / r_{0}$, and substituting geometric constants of the Timoshenko beam and Tersoff-Brenner potential parameters into Eq. (2), the nonlinear constitutive relation of the equivalent beam for a $\mathrm{C}-\mathrm{C}$ bond can be derived as

$$
\sigma=20.852\left(-e^{-4.685 \varepsilon}+e^{-3.817 \varepsilon}\right)
$$

The stress-strain curve of the equivalent beam is also depicted in Fig. 1.

\subsection{Numerical examples and verifications}

The mechanical properties and dynamic fracture of graphene model are simulated in ABAQUS/Explicit which is an explicit dynamic solver based on central difference method [19], and the 2D FE models are constructed by Beam21 (shear) and Mass elements. In our model of graphene, a node at either end of beam element represents a carbon atom, while the beam only reflects the reaction between nodes. In order to simulate the real system of $\mathrm{C}-\mathrm{C}$ bonds and dynamic effects as far as possible, approximately $99 \%$ mass of carbon atoms is deployed at nodes. Equivalent Young's modulus $E$ and Poisson's ratio $\mu$ of the graphene FE models are examined by verification samples under uniaxial tension along ZZ and AC directions. The sample model consists of 14490 nodes and 21565 beams with its width $H=190.3 \AA$ and length $L=196.8 \AA$, shown in Fig. 2 , where $\mathrm{ZZ}$ and $\mathrm{AC}$ edges are along the $X$ and $Y$ axes respectively. The material constitutive relation of beam is coded as VUMAT subroutine in ABAQUS/Explicit, and the VUMAT will be called to update stress tensor and other variables for material calculation points of the beam element within each increment. When material points satisfy the failure criterion (axial stress of B21 element) defined according to $r_{c}$, the beams (atomic bonds) will be removed from the model so that the dynamic fracture process of the model is fulfilled. Only the valid material points are returned at the end of each increment.

To obtain the mechanical properties of graphene model, uniform normal loadings of displacement $\Delta L=13.0 \AA$ are applied gradually to nodes on one edge step by step in Fig. 2, and the other edge is fixed. All the simulations are carried out quasi-statically [19], and explicit marches a solution forward with possible small time increment, which is similar to

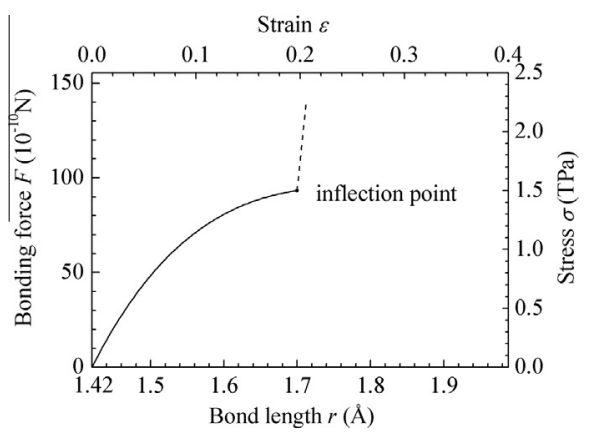

Fig. 1. The constitutive relation of the equivalent beam for $\mathrm{C}-\mathrm{C}$ bond according to the Tersoff-Brenner potential truncated after the inflection point (dash line) to avoid unphysical spurious bond forces. 


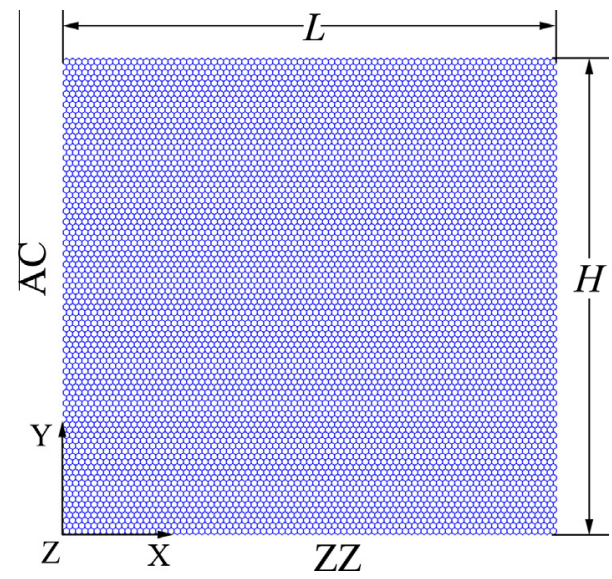

Fig. 2. The FE model of pristine graphene with a width $H$ and a length $L$. Tensile deformations are imposed along AC $(Y)$ or $Z Z(X)$ direction.

velocity-Verlet integration algorithm in MD simulations [5]. The nodal coordinates of the carbon atoms are perturbed at each time increment.

The stress-strain curves during tensile deformations along ZZ and AC orientations are obtained, which falls within the ranges of experimental results [1] in Fig. 3(a). Moreover, the latest study conducted by density functional theory calculations [28] indicates that the stress-strain curves overlap each other under small strains $<15 \%$, and ZZ edge graphene strip is stiffer than AC graphene under strains $>15 \%$, which are agreed with our results despite of overlapping under smaller strains $<4 \%$. The obtained Poisson's ratios are also close to the experimental value [29] under small strains in Fig. 3(b).

\subsection{Fracture model of graphene}

The geometric parameters of graphene ribbon are chosen to match the strain rates in our previous MD simulations [5] for comparisons. A strip model with an initially straight crack (five bonds removed) is constructed with a size of $l \times h=491.9 \AA \times 49.7 \AA$, in which 9624 nodes (i.e. atoms) and 14207 beam elements (i.e. bonds) are used, shown in Fig. 4. As cracks along ZZ edges in graphene usually propagate self-similarly [30], only ZZ crack models are studied here. The original crack will grow under pure opening load of displacement $\Delta h$ displaced away at strain rate $\dot{\varepsilon}=\Delta h /\left(h \cdot t_{t}\right)$. The thickness $t_{g}$ of graphene is assumed to be $3.34 \AA ̊$ under plane stress condition. The deformation-control method is implemented by applying uniform increment $\Delta h / 2$ of $Y$-displacement (less than $0.25 \times 10^{-3} \AA$ per step) gradually to the top and bottom nodes, while $X$-displacement of a bottom node at the middle of the model is constrained and its adjacent B21 elements are clamped. Five strain rates, $\dot{\varepsilon}=1.959 \times 10^{-5} \mathrm{fs}^{-1}, 9.796 \times 10^{-6} \mathrm{fs}^{-1}, 4.898 \times 10^{-6} \mathrm{fs}^{-1}, 9.796 \times 10^{-7} \mathrm{fs}^{-1}$ and $_{9.796 \times 10^{-8}} \mathrm{fs}^{-1} \mathrm{respec}^{-}$ tively, are imposed, and the total time periods $t_{t}$ for analysis are $8 \mathrm{ps}$ (equals the total loading period of the model), $16 \mathrm{ps,}$ $32 \mathrm{ps}, 160 \mathrm{ps}$ and $1600 \mathrm{ps}$ respectively, and the uniform time increment is $0.25 \mathrm{fs}$.

Here time increment in ABAQUS/Explicit algorithm (central difference method) is small enough to guarantee the convergence during the whole process of model fracture. Firstly, time increment is automatically estimated and changed by ABAQUS/Explicit for trial calculations. Then we select the minimum $0.25 \mathrm{fs}$ among these time increments as the fixed value for new calculations at every strain rates. The damping parameters are not defined here for ideal condition.
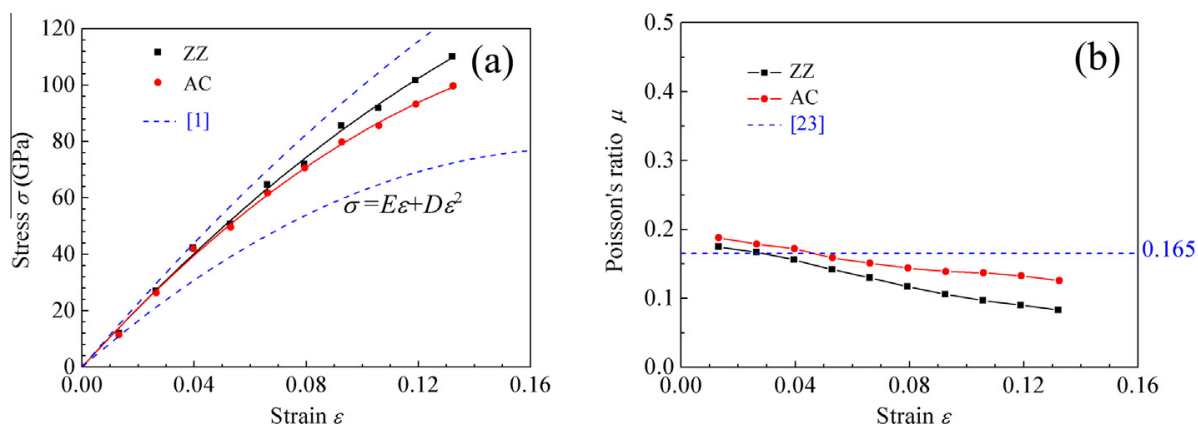

Fig. 3. Pristine grapheme model under tensile loading applied in AC and $\mathrm{ZZ}$ directions, respectively. (a) The stress-strain curves, comparing with experimental results (dot lines) $\sigma=E \varepsilon+D \varepsilon^{2}(E=1018 \pm 150 \mathrm{GPa}$ and $D=2066 \pm 359 \mathrm{GPa}$, normalized by thickness $3.34 \AA$ ) [1]; (b) Poisson's ratio vs. strain, comparing with 0.165 (dot line) [29]. 


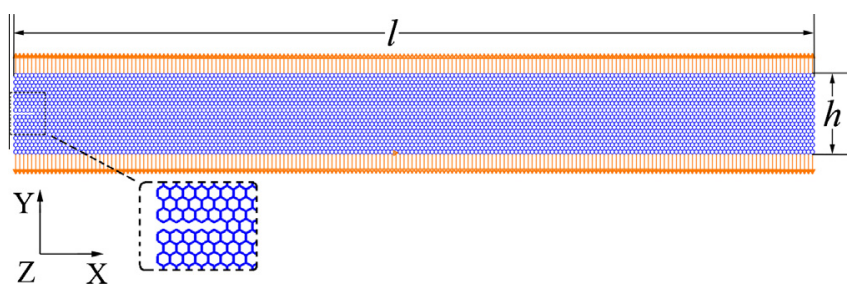

Fig. 4. The initial FE strip model of graphene (blue lattice) under uniform opening loads of displacements (depicted by orange arrows on both top and bottom nodes) in $Y$ direction perpendicular to the crack line. The strip model has a width $h$ and a length $l$ with an initially straight crack by removing five bonds (snapshot). (For interpretation of the references to colour in this figure legend, the reader is referred to the web version of this article.)

(a)

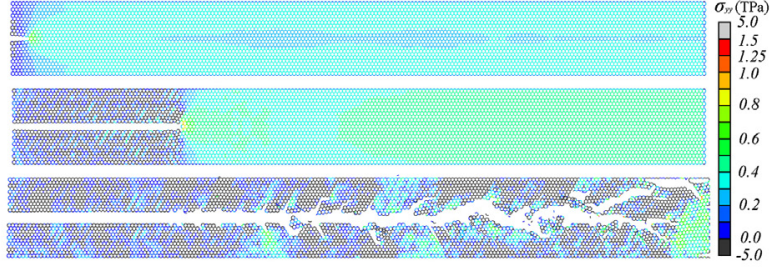

(b)

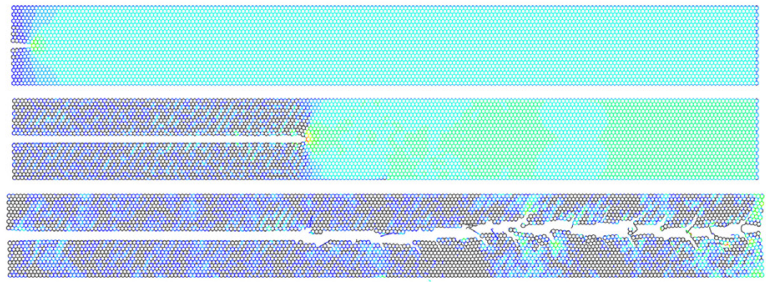

(c)

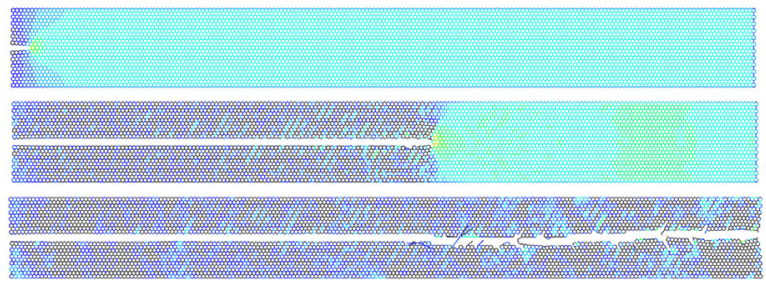

(d)

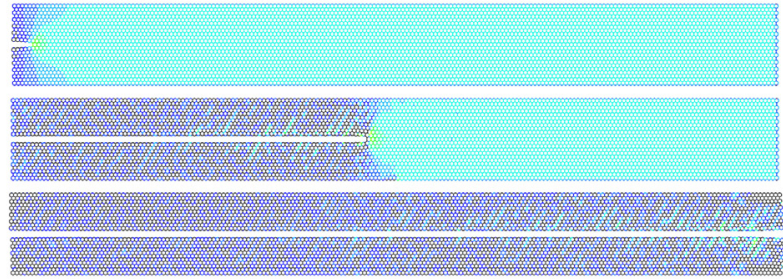

(e)

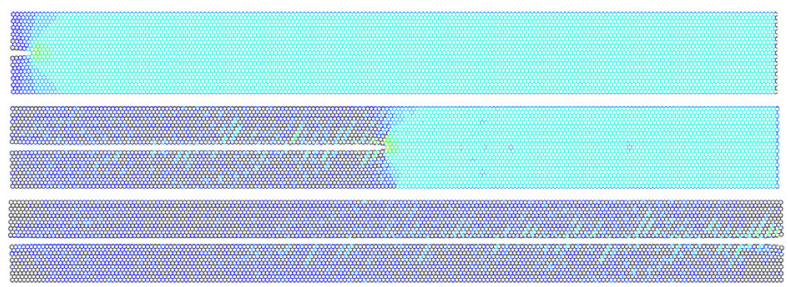

Fig. 5. Stress contours of crack propagation at different stages (a-e): initiation (top); propagation (middle); final failure (bottom). (a-c) crack branching, (de) straightforward fracture. Strain rate $\dot{\varepsilon}=$ (a) $1.959 \times 10^{-5} \mathrm{fs}^{-1}$; (b) $9.796 \times 10^{-6} \mathrm{fs}^{-1}$; (c) $4.898 \times 10^{-6} \mathrm{fs}^{-1}$; (d) $9.796 \times 10^{-7} \mathrm{fs}^{-1} \mathrm{and}^{(\mathrm{e})} 9.796 \times 10^{-8} \mathrm{fs}^{-1}$. The colour scale bar on the right side of (a) represents the magnitudes of stresses $\sigma_{y y}$, which is applicable to all contours. 


\section{Results and discussions}

\subsection{The characteristics of dynamic fracture}

The dynamic fracture of the graphene FE models is illustrated in Fig. 5. As the displacements of the top and bottom edges increase bit by bit, energy from external work flows from the border to the centre of the model. At the same time, beam elements of the whole model are tensed, and stress concentrates quickly around crack tips. Initial crack propagates instantly after the breakage of $\mathrm{C}-\mathrm{C}$ bond reaching the critical length $r_{\mathrm{c}}$ at the crack fronts.

The position of crack tip is determined by finding the final breakage of the $\mathrm{C}-\mathrm{C}$ bond ahead. The velocity $V=\Delta a / \Delta t$ at every crack length $a$ is measured over relevant distance $\Delta a$ just before the crack tip within a time interval $\Delta t$. The distances used to calculate $V$ are selected every ten lattices (lattice spacing $2.46 \AA$ ) at smooth crack edges/surfaces or every five lattices densely at irregular edges/surfaces. Combining with Fig. 5, we can observe that $V$ increases sharply within a short distance once the crack is initiated, then reaches a relatively stable value as crack grows straight and smoothly in a "mirror" style. The cracks in all cases move forward, and the first three in Fig. 6(a)-(c) begin to kink and oscillate at higher strain rates after propagation of different distances, while cracks keep straight in Fig. 6(d)-(e) at lower rates.

As shown in Fig. 6(a)-(c), instabilities appear and the velocities fluctuate significantly once the kinking occurs. Then, the cracks tend to deviate from their straight paths and the crack edges become more irregular accompanied with oscillatory overhangs, topological defects, and carbon chains (beams) bridging. The cracks under lower strain rates move straight, and the velocities remain almost unchanged in Fig. 6(d)-(e).
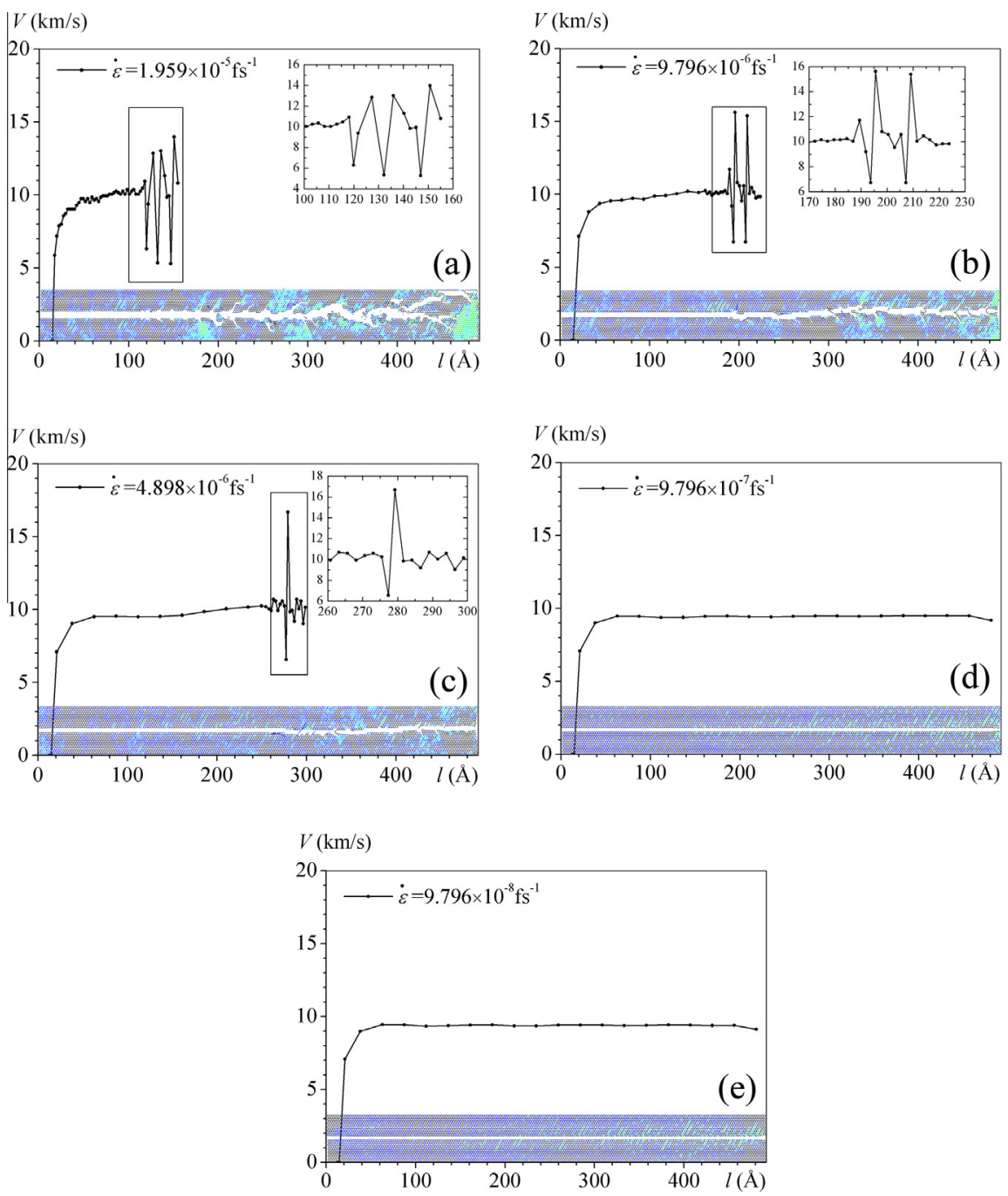

Fig. 6. Velocity $V$ of crack motion in graphene. Straight cracks start to kink when $V$ is beyond $10.33 \mathrm{~km} / \mathrm{s}$, about $82 \%$ of the Rayleigh-wave speed ( $12.56 \mathrm{~km} /$ s), which can be observed in the morphologies of fracture edges in (a)-(c) accompanied with oscillations of velocity (zooming in), while cracks undergo along straight lines enduringly without branching/kinking at lower velocities in (d)-(e). 
(a)

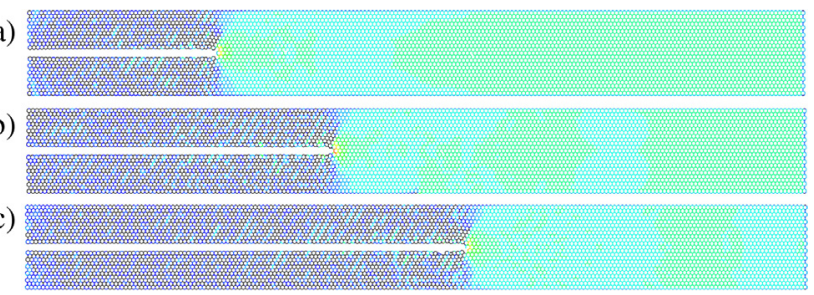

Fig. 7. The stress contours of the whole models at the moments just before the first kinking/branching under three strain rates $\dot{\varepsilon}=(\mathrm{a}) 1.959 \times 10^{-5}$ fs ${ }^{-1}$; $(\mathrm{b})$ $9.796 \times 10^{-6} \mathrm{fs}^{-1} ;$ (c) $4.898 \times 10^{-6} \mathrm{fs}^{-1}$.

Table 2

The strain $\varepsilon$ and critical SIF $K_{C}$, velocity $V_{C}$, energy release rate $S_{C}$, average velocity $\bar{V}$ at crack initiating, kinking and completely ending at five strain rates.

\begin{tabular}{|c|c|c|c|c|c|c|c|}
\hline \multirow[t]{2}{*}{ Strain rate $\dot{\varepsilon}\left(\mathrm{fs}^{-1}\right)$} & \multicolumn{3}{|l|}{ Strain $\varepsilon$} & \multirow[t]{2}{*}{$K_{C}(\mathrm{MPa} \sqrt{ } \mathrm{m})$} & \multirow[t]{2}{*}{$V_{C}(\mathrm{~km} / \mathrm{s})$} & \multirow[t]{2}{*}{$S_{C}(\mathrm{TPa} \AA)$} & \multirow[t]{2}{*}{$\bar{V}(\mathrm{~km} / \mathrm{s})$} \\
\hline & Initiating (\%) & Kinking (\%) & Ending (\%) & & & & \\
\hline $1.959 \times 10^{-5}$ & 4.99 & 7.17 & 13.40 & 3.70 & 10.42 & 0.131 & 11.11 \\
\hline $9.796 \times 10^{-6}$ & 5.07 & 6.90 & 9.66 & 3.59 & 10.30 & 0.123 & 10.09 \\
\hline $4.898 \times 10^{-6}$ & 5.11 & 6.45 & 7.52 & 3.34 & 10.28 & 0.107 & 9.69 \\
\hline $4.898 \times 10^{-6}$ & 4.14 & 4.68 & 8.72 & 3.59 & 9.94 & 0.123 & 10.36 \\
\hline $9.796 \times 10^{-7}$ & 5.14 & - & 5.64 & - & - & - & 9.33 \\
\hline $9.796 \times 10^{-8}$ & 5.15 & - & 5.20 & - & - & - & 9.30 \\
\hline
\end{tabular}

${ }^{\text {a }}$ Larger model $l \times h=983.81 \AA \times 100.82 \AA$.

The crack kinking occurs earlier at higher strain rates, while later at lower strain rates in Fig. 7, and even vanishes in Fig. 6(d)-(e) where the crack speeds cannot reach the threshold value. The initial strains are smaller than the results in [8], and decrease slightly with the increase of strain rate, which is different from [8]. Critical strain $\varepsilon_{C}$ is calculated by $\varepsilon_{C}=\left(t_{k} \cdot \Delta h\right) /\left(t_{t} \cdot h\right)$, where $t_{k}$ is the time for crack kinking. Critical strains are $7.17 \%, 6.90 \%$ and $6.45 \%$ respectively at high strain rates, as shown in Table 2.

\subsection{Analysis of SIF, energy release rate and velocity}

The critical SIFs of graphene when crack kinking occurs under three strain rates are calculated by $K_{C}=\sqrt{2 /\left(h-\mu^{\prime 2} h\right)} E^{\prime} \Delta h_{c}$ (where $\Delta h_{C}=0.5 h \varepsilon_{C}$, Young's modulus $E^{\prime}=1.025$ TPa and Poisson's ratio $\mu^{\prime}=0.165$ for graphene) [5], yields $K_{C}=3.70,3.59$ and $3.34 \mathrm{MPa} \sqrt{ } \mathrm{m}$ at critical strains $\varepsilon_{C}=7.17 \%, 6.90 \%$ and $6.45 \%$ respectively, as shown in Table 2 and Fig. 7(a)-(c). Compared with reported data $3.16 \mathrm{MPa} \sqrt{ } \mathrm{m}[30]$ and $4.21 \mathrm{MPa} \sqrt{ } \mathrm{m}[10]$, our results are reasonable.

As the crack motion is triggered after the first bond breakage, the potential energy stored around the crack tip is released and transformed into surface energy or atomic vibration energy. The crack continues to grow with external energy flowing into crack tip field and forming fresh surfaces with enough surface energy. The energy release rates $S_{C}=0.5 h \varepsilon_{C}^{2} E^{\prime}\left(\left(E^{\prime}-\left(1-\mu^{\prime 2}\right) V_{C}^{2} \rho\right) /\left(E^{\prime}-V_{C}^{2} \rho\right)\right)$ [31] are then obtained as $0.131,0.123$ and 0.107 TPa $\AA$, which are very close to $\mathrm{MD}$ results $0.130,0.119$ and $0.125 \mathrm{TPa} \AA$ respectively. The energy release rate at crack tip is reasonably larger than the energy cost of breaking a graphene sheet, i.e. the surface energy $2 \gamma=1.0997 \mathrm{TPa} \AA$ (averaged over thickness $t_{g}=3.34 \AA$ ), indicating that energy dissipation is considerable here.

The critical velocity of crack instability $V_{C}$ is obtained by averaging over possible small interval of crack increment before occurrences of kinking in Fig. 6(a)-(c). The average velocities at five strain rates are calculated by $\bar{V}=\Delta a / \Delta t$ (where

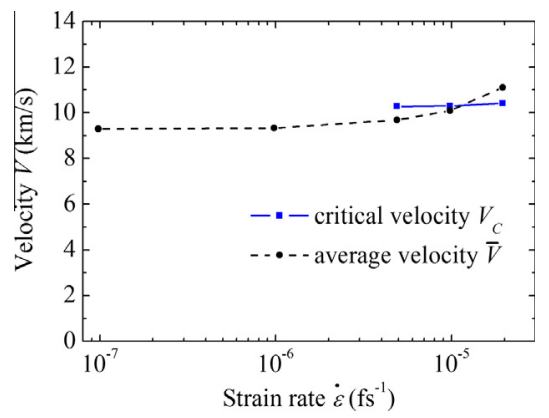

Fig. 8. The velocity of crack motion changes with strain rates. 
$\Delta a=477.15 \AA$ is the whole length of the crack, and $\Delta t$ is the corresponding total time interval), which yields $11.11,10.09$, $9.69,9.33$ and $9.30 \mathrm{~km} / \mathrm{s}$ correspondingly. All the five values are relatively larger than those in [5]. These differences may be due to the different intrinsic mechanism between continuum models and MD ones. $\bar{V}$ increases with strain rates (loading rates), while $V_{C}$ remains $10.33 \mathrm{~km} / \mathrm{s}$ approximately in Fig. 8, which confirms that a threshold velocity of crack instability does exist. By use of the phase velocity of Raylaigh-wave $V_{r}=12.56 \mathrm{~km} / \mathrm{s}$ [5], $V_{C} \approx 0.82 V_{r}$ appears at three high strain rates, which is larger than Yoffe's theoretical predictions $\sim 0.73 V_{r}$ [6] and Zhang's MD result $\sim 0.65 V_{r}$ [5]. Our results of larger FE model of graphene show that the initial strain, critical strain and critical velocity are a little smaller than those of small model, as shown in Table 2, these differences should be considered in further giant-scale parallel calculations.

\section{Conclusions}

We have studied the velocities and instabilities of moving cracks in equivalent FE models of graphene strips under mode-I displacement loading, in which $\mathrm{C}-\mathrm{C}$ bonds are represented by Timoshenko beam elements. The nonlinear constitutive relation of beams is derived from the modified reactive empirical bond-order potential of carbon. The cracks propagate straight along zigzag direction supersonically under low strain rates $9.796 \times 10^{-7} \mathrm{fs}^{-1}$ to $9.796 \times 10^{-8} \mathrm{fs}^{-1}$. While under high strain rates $1.959 \times 10^{-5} \mathrm{fs}^{-1}$ to $4.898 \times 10^{-6} \mathrm{fs}^{-1}$, the cracks kink beyond a critical velocity around $10.33 \mathrm{~km} / \mathrm{s}$ equivalent to 0.82 $V_{r}$ (Rayleigh-wave speed in graphene), which is comparable with the results of theoretical predictions and MD simulations. Thus our FE model would be potential to investigate the rapid fracture behaviours of 2D hexagonal crystals.

\section{Acknowledgements}

This work is partially supported by NSFCs (11372131, 11232007 and 11172130), Aeronautical Science Foundation (2012ZF52074), State Key Laboratory of Mechanics and Control of Mechanical Structures (NUAA, No. MCMS-0114G01; MCMS-0215G01), 973 Program (2011CB707602), the Fundamental Research Funds for the Central Universities and the PAPD of China. X.L. is also supported by NSFC (11202214) and 973 Program of China (2012CB937500). B.Z. also thanks for Newton International Fellowship (NF080039) and Newton Alumni Follow-On of UK's Royal Society hosted by University of Glasgow and Newcastle University.

\section{References}

[1] Lee C, Wei X, Kysar W, Hone J. Measurement of the elastic properties and intrinsic strength of monolayer graphene. Science 2008;321:385-8.

[2] Zhang B. Layered graphene structure of a hexagonal carbon. Phys B 2013;418:73-5.

[3] Rafiee MA, Rafiee J, Srivastava I, Wang Z, Song H, Yu Z, et al. Fracture and fatigue in graphenenanocomposites. Small 2010;6(2):179-83.

[4] Le MQ, Batra RC. Single-edge crack growth in graphene sheets under tension. Comput Mater Sci 2013;69:381-8.

[5] Zhang B, Yang G, Xu H. Instability of supersonic crack in graphene. Phys B 2014;434:145-8.

[6] Yoffe EH. LXXV. The moving griffith crack. Philos Mag 1951;42(330):739-50.

[7] Omeltchenko A, Yu J, Kalia RK, Vashishta P. Crack front propagation and fracture in a graphite sheet: a molecular-dynamics study on parallel computers. Phys Rev Lett 1997;78(11):2148.

[8] Wang C, Han Q, Xin D. Fracture analysis of single-layer graphene sheets with edge crack under tension. Mol Simulat 2014:1-8 (ahead-of-print).

[9] Zhao H, Aluru N. Temperature and strain-rate dependent fracture strength of graphene. J Appl Phys 2010;108(6):064321.

[10] Xu M, Tabarraei A, Paci JT, Oswald J, Belytschko T. A coupled quantum/continuum mechanics study of graphene fracture. Int J Fracture 2012;173(2):163-73.

[11] Kim K, Artyukhov VI, Regan W, Liu Y, Crommie MF, Yakobson BI, et al. Ripping graphene: preferred directions. Nano Lett 2011;12(1):293-7.

[12] Lee JH, Loya PE, Lou J, Thomas EL. Dynamic mechanical behavior of multilayer graphene via supersonic projectile penetration. Science 2014:346(6213):1092-6.

[13] Li C, Chou TW. A structural mechanics approach for the analysis of carbon nanotubes. Int J Solids Struct 2003;40(10):2487-99.

[14] Tserpes KI, Papanikos P, Tsirkas SA. A progressive fracture model for carbon nanotubes. Composites Part B 2006;37(7):662-9.

[15] Baykasoglu C, Mugan A. Nonlinear fracture analysis of single-layer graphene sheets. Eng Fract Mech 2012;96:241-50.

[16] Mohammadpour E, Awang M. Nonlinear finite-element modeling of graphene and single-and multi-walled carbon nanotubes under axial tension. Appl Phys A 2012;106(3):581-8.

[17] Parashar A, Mertiny P. Study of mode I fracture of graphene sheets using atomistic based finite element modeling and virtual crack closure technique. Int J Fract 2012;176:119-26.

[18] Scarpa F, Adhikari S, Phani AS. Effective elastic mechanical properties of single layer graphene sheets. Nanotechnology 2009;20(6):065709.

[19] Hibbit KAS. ABAQUS Theory and User Manuals Version 6.9. USA: ABAQUS Inc.; 2009.

[20] Belytschko T, Xiao SP, Schatz GC, Ruoff RS. Atomistic simulations of nanotube fracture. Phys Rev B 2002;65(23):235430.

[21] Jorgensen WL, Severance DL. Aromatic-aromatic interactions: free energy profiles for the benzene dimer in water, chloroform, and liquid benzene. J Am Chem Soc 1990;112(12):4768-74.

[22] Chandra Y, Scarpa F, Chowdhury R, Adhikari S, Sienz J. Multiscale hybrid atomistic-FE approach for the nonlinear tensile behaviour of graphene nanocomposites. Composites Part A 2013;46:147-53.

[23] Dumitrică T, Belytschko T, Yakobson BI. Bond-breaking bifurcation states in carbon nanotube fracture. J Chem Phys 2003;118(21):9485-8.

[24] Lu Q, Bhattacharya B. The role of atomistic simulations in probing the small-scale aspects of fracture-a case study on a single-walled carbon nanotube. Eng Fract Mech 2005;72(13):2037-71.

[25] Brenner DW. Empirical potential for hydrocarbons for use in simulating the chemical vapor deposition of diamond films. Phys Rev B 1990;42(15):9458.

[26] Brenner DW, Shenderova OA, Harrison JA, Stuart SJ, Ni B, Sinnott SB. A second-generation reactive empirical bond order (REBO) potential energy expression for hydrocarbons. J Phys: Condens Matter 2002;14(4):783.

[27] Huang Y, Wu J, Hwang KC. Thickness of graphene and single-wall carbon nanotubes. Phys Rev B 2006;74(24):245413.

[28] Yin H, Qi HJ, Fan F, Zhu T, Wang B, Wei Y. Griffith criterion for brittle fracture in graphene. Nano Lett 2015. http://dx.doi.org/10.1021/nl5047686.

[29] Blakslee OL, Proctor DG, Seldin EJ, Spence GB, Weng T. Elastic constants of compression-annealed pyrolytic graphite. J Appl Phys 1970;41:3373.

[30] Zhang B, Mei L, Xiao H. Nanofracture in graphene under complex mechanical stresses. Appl Phys Lett 2012;101(12):121915.

[31] Freund LB. Dynamic fracture mechanics. Cambridge University Press; 1998. 